\title{
How-I-Do-It
}

\section{Anterior pancreatic duct split prior to duct-to-mucosa pancreatico-jejunal anastomosis in pancreaticoduodenectomy}

\author{
Srikanth Gadiyaram, Murugappan Nachiappan
}

Department of Surgical Gastroenterology and Minimally Invasive Surgery, Sahasra Hospitals, Bangalore, India

\begin{abstract}
A secure pancreatic-enteric anastomosis is widely accepted as the 'Achilles heel' in reconstruction following a pancreaticoduodenectomy. Most morbidity following the procedure is related to the failure of this anastomosis, resulting in intra-abdominal collections, secondary haemorrhage, delayed gastric emptying, need for radiological interventions and re-operation for some patients. Of several techniques available, the 'duct-to-mucosa' technique is widely employed for pancreaticojejunal anastomosis. Among several refinements to facilitate this anastomosis, viz; mobilization of pancreatic stump, magnification with loupes and modifications made on the jejunal side to enable a tension free anastomosis, none seems to address the pancreatic duct in particular. The operative technique of anterior pancreatic duct split described by us enables a wider, well visualized pancreatic duct for a secure duct to mucosa pancreaticojejunal anastomosis.
\end{abstract}

Key Words: Pancreas; Pancreaticoduodenectomy; Pancreaticojejunostomy; Duct-to-mucosa anastomosis; Anterior pancreatic duct split

\section{INTRODUCTION}

A secure pancreatic-enteric anastomosis is widely accepted as the 'Achilles heel' in reconstruction following pancreaticoduodenectomy. Most morbidity following the procedure is related to the failure of this anastomosis, resulting in intra-abdominal collections, secondary haemorrhage, delayed gastric emptying, need for radiological interventions and re-operation for some patients [1]. Innumerable techniques described for reconstruction are proof of the lack of uniform good results with one particular technique when it is adopted by others [2]. We herein describe our technique of anterior pancreatic duct split (APDS) to facilitate a wide duct-to-mucosa pancreaticojejunostomy (PJ) as part of reconstruction following pancreaticoduodenectomy.

Received: May 18, 2021, Accepted: June 7, 2021

Corresponding author: Srikanth Gadiyaram Department of Surgical Gastroenterology and Minimally Invasive Surgery, Sahasra Hospitals, New no 30, 39th Cross,Jayanagar 8th Block, Bangalore 560082 , India

Tel: +91-9880109971, E-mail: srikanthgastro@gmail.com

ORCID: https://orcid.org/0000-0001-6676-2030

Copyright (C) The Korean Association of Hepato-Biliary-Pancreatic Surgery

\section{DESCRIPTION OF SURGICAL TECHNIQUE}

After completing pancreaticoduodenal resection, hemostasis is ensured. Stay sutures previously placed at cranial and caudal transected ends of the pancreatic stump are gently retracted to the left in order to view the pancreatic duct (PD). A Gerald type forceps with closed prongs is advanced into the PD for a length of a few $\mathrm{mm}$ and gently opened in a horizontal plane. Using a needle tip cautery with a cutting current, the parenchyma and the PD in anterior mid-portion are progressively cut. A fine metal suction is used to suck out any blood in the field. The Gerald forceps is then gently advanced a few more mm into the duct and the APDS is completed for a predetermined length (vide infra) which is equal to the length of the circumference of the PD (Fig. 1). With the forceps in-situ, an apical suture is placed through the duct (inside out) using a double arm 4-0 Polydioxanone (PDS) suture. The PD available for anastomosis after APDS is approximately three times the circumference of the original duct (Fig. 2). The point 'A' after split becomes 'A1' and 'A2'. Stay sutures adjacent to these points with 4-0 PDS double arm serve the purpose of defining the 'corners' for the anterior and posterior layers. The entire circumference of the PD and the cut surface of the transected pancreas at neck region prior to the APDS become part of the posterior wall, 


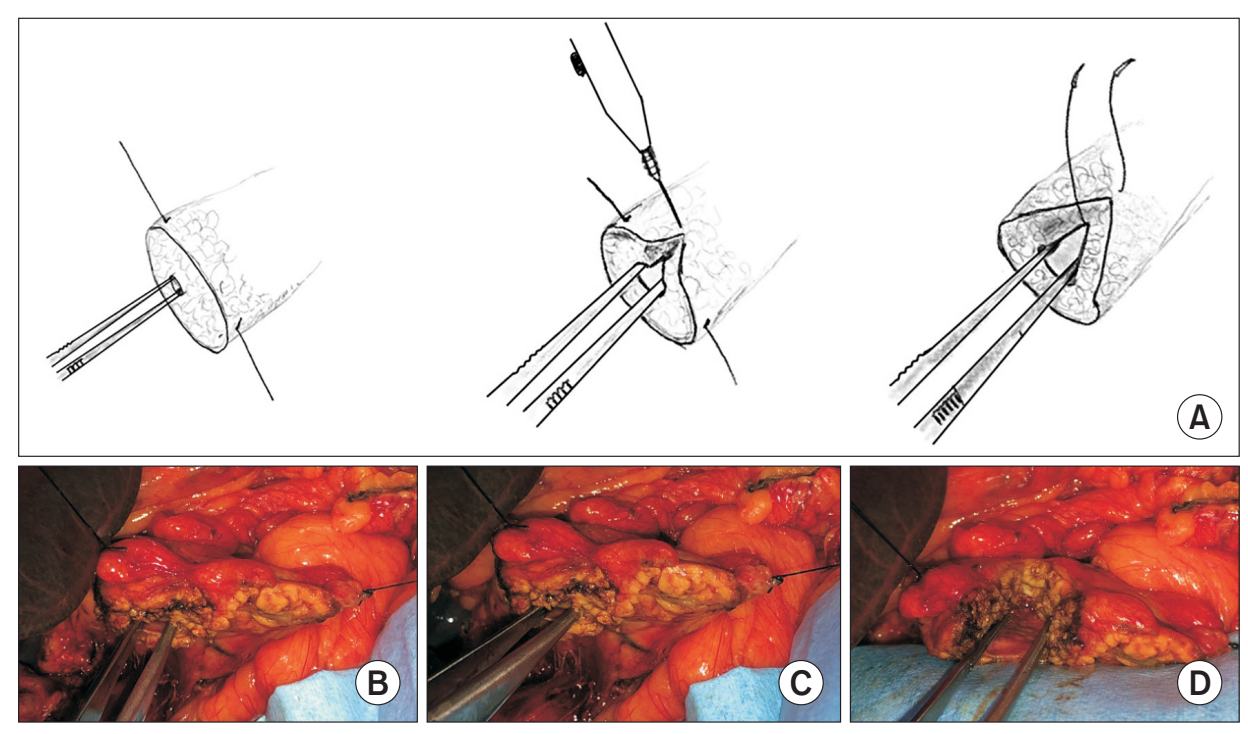

(A)

while the part of the PD along with the adjacent cut open area of the pancreas exposed by the APDS becomes the anterior wall for the planned PJ. A double arm 4-0 PDS posterior row of sutures on the PD are placed 'inside out' at a distance of 2 $\mathrm{mm}$ from each other, exiting from the pancreatic capsule at 3-4 $\mathrm{mm}$ between each suture to adjust for the greater length on the capsular edge of the wall (Fig. 3). These sutures are serially held with 'shods'. The jejunal end is brought adjacent to the pancreatic stump. An interrupted row of 3-0 silk sutures as capsular stitches are placed on the pancreas at about 4 to $5 \mathrm{~mm}$ from the edge and seromuscular on the jejunum to anchor the jejunum to the pancreas. A jejunotomy of appropriate length is made horizontally, opposite the PD. The corner sutures are passed on the jejunal side inside-out and held with shods. The posterior row of preplaced sutures on the PD side are now serially passed (outside-in) on the jejunal side posteriorly. The posterior row of sutures are serially tied, completing the posterior layer of the anastomosis (Fig. 4). The anterior row of sutures are then preplaced at $2 \mathrm{~mm}$ interval on the PD side as an initial step, again 'inside out'. The inner ends of the double arm sutures are subsequently serially passed into the anterior lip of the jejunum (inside-out). The anterior row of sutures are tied to complete the anterior layer of the duct-to-mucosa anastomosis (Fig. 5). The hepaticojejunostomy and gastrojejunostomy are done in the usual manner. Two drains are placed. One is placed adjacent to PJ anastomosis while the other is placed behind the biliary enteric anastomosis.

\section{DISCUSSION}

A secure duct-to-mucosa PJ needs to meet three essential criteria. Firstly, sutures need to be carefully placed on the PD and the jejunal mucosal side at an appropriate distance. Secondly, vascularity of pancreatic and jejunal sides need to be good. 'A' after the split.
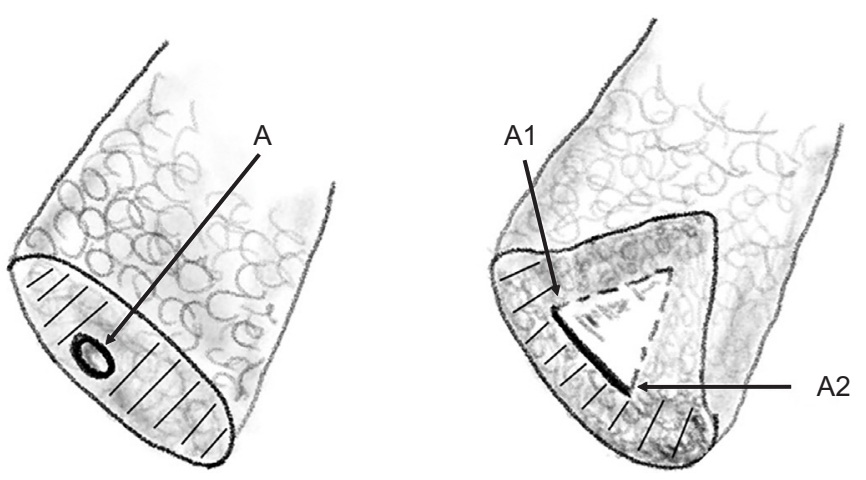

Fig. 1. (A) Illustrations depicting the anterior pancreatic ductal split (APDS). (B) Operative photograph showing the pancreatic duct. (C) Initial APDS. (D) Completed APDS.

\begin{tabular}{|c|c|c|c|}
\multicolumn{4}{|c|}{ Circumference of pancreatic duct (bold line) $=\pi \mathrm{d}$} \\
\hline Diameter & Circumference (C) & APDS & $\begin{array}{c}\text { Total length of PD } \\
\text { available } 3^{*}(C)\end{array}$ \\
\hline $2 \mathrm{~mm}$ & $6.3 \mathrm{~mm}$ & $6.3 \mathrm{~mm}$ & $18.8 \mathrm{~mm}$ \\
\hline $3 \mathrm{~mm}$ & $9.4 \mathrm{~mm}$ & $9.4 \mathrm{~mm}$ & $28.3 \mathrm{~mm}$ \\
\hline $4 \mathrm{~mm}$ & $12.6 \mathrm{~mm}$ & $12.6 \mathrm{~mm}$ & $37.7 \mathrm{~mm}$ \\
\hline
\end{tabular}

Fig. 2. Illustrations showing the entire circumference of the pancreatic duct (PD) prior to anterior pancreatic duct split (APDS) forming the posterior layer (bold line) of the duct for anastomosis and the entire transected surface of the pancreas prior to the split forming the posterior wall of the anastomosis (shaded area). Extra length of the duct available for the anastomosis post APDS is represented by dashed lines. ' $A$ ' point, where the duct is split; ' $A 1$ ' and 'A2' points correspond to point

Thirdly, the anastomosis needs to be tension free [3].

Our technique described herein started with the background of the senior author (SG) having performed over 200 pancreaticoduodenectomies with the 'duct-to-mucosa' technique for pancreaticojejunal anastomosis. The PD is dilated in more than $80 \%$ of patients with pancreatic head or periampullary 
A
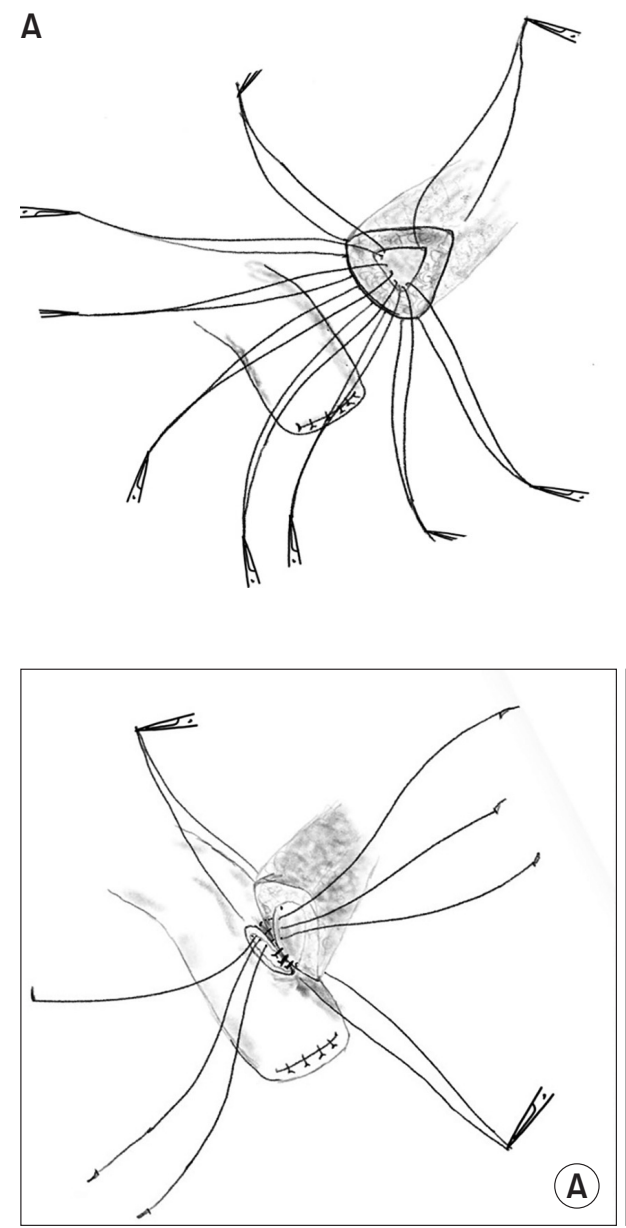

B

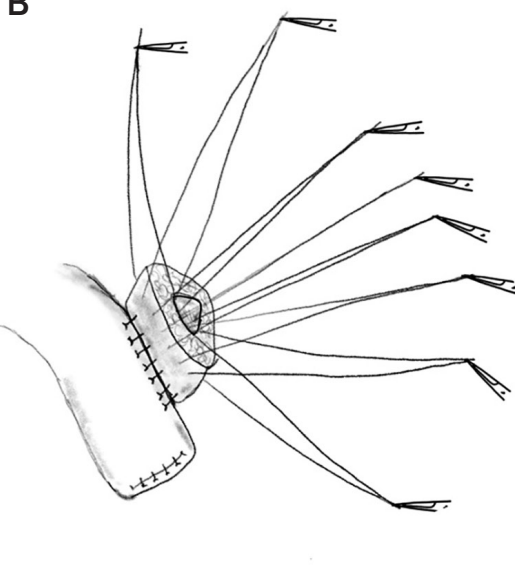

Fig. 3. (A) Illustration depicting pre-placed sutures on the posterior layer of the pancreatic duct. Note that this involves the entire circumference of the duct and the cut surface of the pancreas prior to the split. (B) Anchoring sutures on the jejunum.

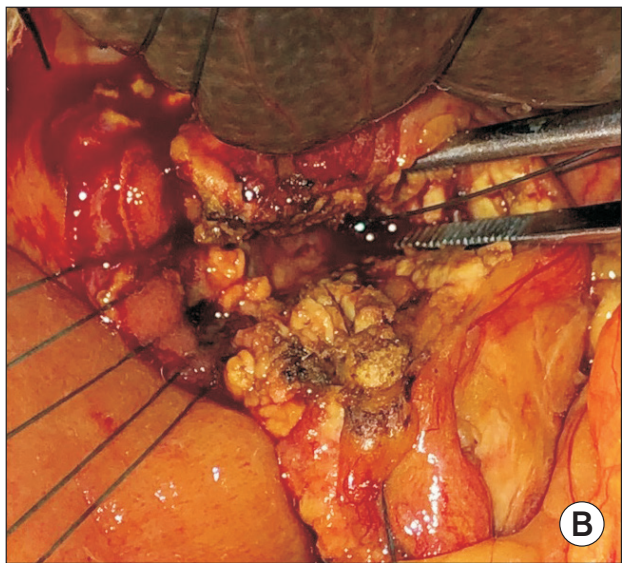

Fig. 4. (A) Illustration showing the posterior layer pre-placed sutures being tied. (B) Operative photograph of completed posterior layer of duct-to-mucosa anastomosis. carcinoma [4]. The normal PD is $2 \mathrm{~mm}$ in the body of the pancreas. We have explicated our technique which is applicable to the vast majority of patients with normal or dilated PDs. The authors recognise that for a minority of patients with a PD that cannot be cannulated or when the pancreatic stump is soft, bulky/friable, another technique of PJ reconstruction might need to be adopted.

Placement of sutures on the PD side can be challenging, particularly when the duct is undilated. The PD is seen enface in a vertically transected pancreatic stump remnant. Therefore, it is not in direct line of vision of the operating surgeon. Techniques that enable better placement of sutures generally revolve around adequate mobilisation of the pancreatic stump and the use of loupes for magnification. None of these measures ensures a wider, enface visible duct for equidistant placement of sutures.

As an example, for a PD that is $3 \mathrm{~mm}$ in diameter, the length of duct mucosa utilised is calculated by the circumference of duct which is $9.4 \mathrm{~mm}$ (circumference $=\pi \mathrm{d}$ ) [5]. It follows that, for a duct of $2 \mathrm{~mm}, 3 \mathrm{~mm}$, or $4 \mathrm{~mm}$, APDS would result in three times this length, i.e., $18.8 \mathrm{~mm}, 28.3 \mathrm{~mm}$, or $37.7 \mathrm{~mm}$, respectively of duct edge available for PJ. The usual duct-to-mu- cosa anastomosis would generally allow for placement of 8 to 10 sutures. Current available techniques do not address the need for making a wider duct to ensure a safe anastomosis. Also, the course of $\mathrm{PD}$ is in a more horizontal plane in the body region while it dips steeply near neck region as it enters the pancreatic head, resulting in the duct being more posterior in location after transection. During APDS, it is evident that the PD is in a more anterior location where the apical stitch is taken and the parenchyma is relatively thinner anterior to duct in this region. The wider duct thus exposed, which is horizontally disposed in the direct field of vision of the operating surgeon, allows for precise preplacement of anterior or posterior row of sutures at $2 \mathrm{~mm}$ in distance.

The vascularity of the pancreatic stump is critical in avoiding an anastomotic failure [6]. Cutting back on the stump to ensure bleeding from the PD region, avoiding too much of mobilisation of the stump and excessive use of electrocautery for pancreatic neck transection are methods of good surgical practice suggested by others. The placement of sutures in the usual duct-to-mucosa anastomosis, where the disposition of the duct is in a perpendicular plane to the remnant, might additionally cause a compromise on the vascularity by a 'pinch cock effect' 

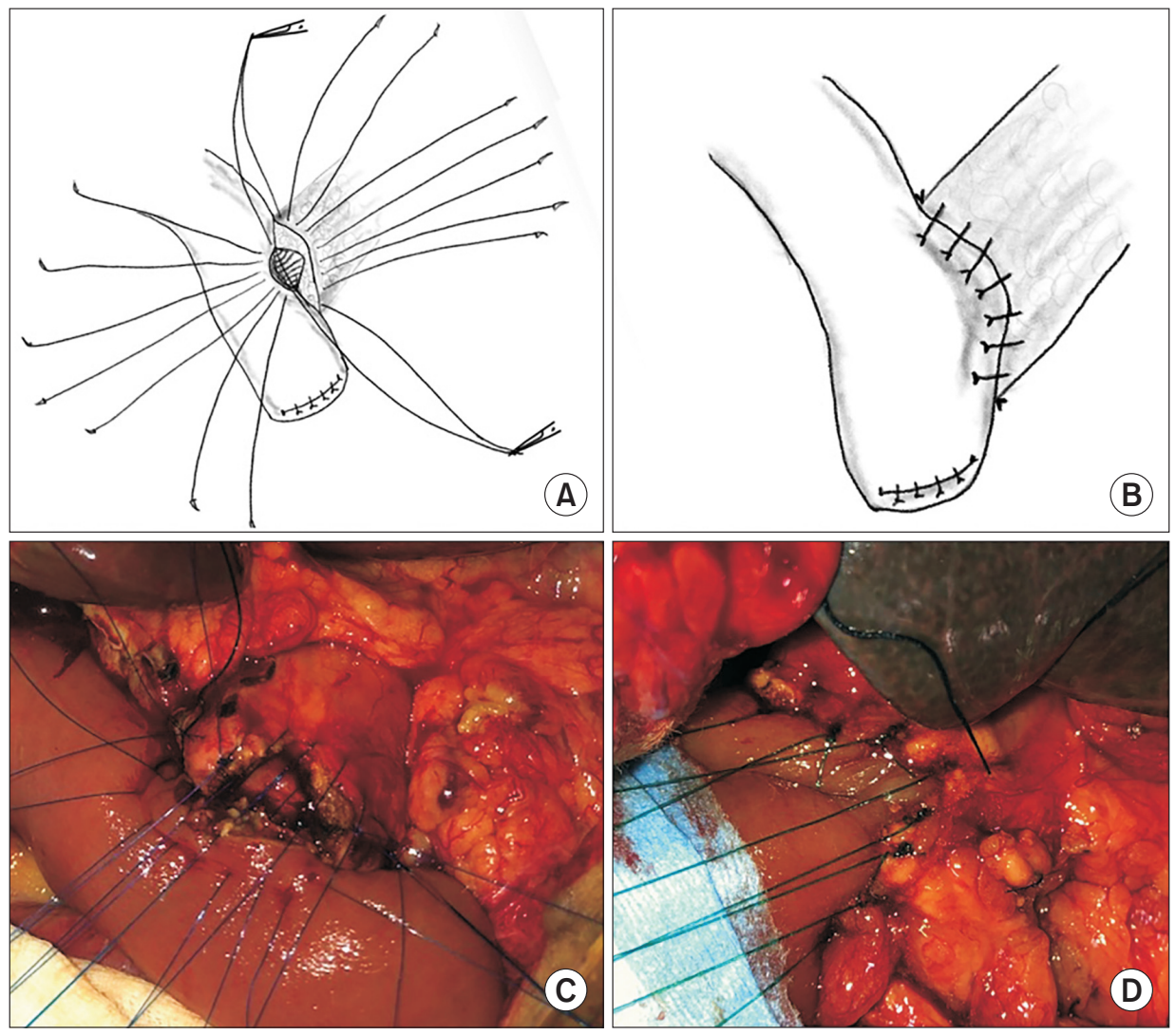

Fig. 5. (A) Illustration showing sutures of the anterior layer of the anastomosis preplaced. (B) The completed duct-to-mucosa anastomosis. (C) Operative photograph of pre-placed sutures of the anterior layer of the duct-to-mucosa anastomosis. (D) Completed anterior layer of the duct-tomucosa anastomosis.

on the edge of the remnant. The APDS ensures an extra length of well-vascularised duct available for anastomosis, thus allowing the jejunal side to 'grab' more of the PD. The vascularity has to be better since APDS is towards a more vascular part of the gland. Bleeding from the parenchyma during APDS can be eminently managed with bipolar or 5-0 polypropylene sutures. However, it would be prudent to place the apical stitch prior to attempts at hemostasis lest there be an occlusion or narrowing of the PD at the apex. Also, gentle traction on the apical stitch and passing a feeding tube into the distal PD can help in placing any hemostatic sutures when required. The greater spread of sutures placed, well-vascularised duct and horizontal disposition of the PJ obviate any 'pinch cock effect' resulting from the usual method of a reconstruction.

In summary, APDS prior to a duct-to-mucosa PJ anastomosis provides several advantages. Firstly, anterior ductotomy of the PD increases the available length for anastomosis. Secondly, it ensures that the anastomosis is done to a well-vascularised PD. Thirdly, it results in a more horizontally disposed duct in the direct line of vision of the operating surgeon. Fourthly, it enables accurate placement of sutures. We believe that APDS allows for a well exposed, wide PD with maintained vascularity. It can be performed for normal or dilated PDs.

\section{CONFLICT OF INTEREST}

No potential conflict of interest relevant to this article was reported.

\section{ORCID}

Srikanth Gadiyaram, https://orcid.org/0000-0001-6676-2030 Murugappan Nachiappan,

https://orcid.org/0000-0001-8687-3096

\section{AUTHOR CONTRIBUTIONS}

Conceptualization: SG. Data curation: MN. Methodology: SG. Visualization: SG, MN. Writing - original draft: SG. Writing - review \& editing: SG, MN.

\section{REFERENCES}

1. Bassi C, Marchegiani G, Dervenis C, Sarr M, Abu Hilal M, Adham M, et al. The 2016 update of the International Study Group (ISGPS) definition and grading of postoperative pancreatic fistula: 11 years after. Surgery 2017;161:584-591.

2. Olakowski M, Grudzińska E, Mrowiec S. Pancreaticojejunostomya review of modern techniques. Langenbecks Arch Surg 2020;405:13- 
22.

3. Shrikhande SV, Qureshi SS, Rajneesh N, Shukla PJ. Pancreatic anastomoses after pancreaticoduodenectomy: do we need further studies? World J Surg 2005;29:1642-1649.

4. Baxi AC, Jiang Q, Hao J, Yang Z, Woods K, Keilin S, et al. The effect of solid pancreatic mass lesions on pancreatic duct diameter at endoscopic ultrasound. Endosc Ultrasound 2017;6:103-108.
5. Perry O, Perry J. The geometry of the circle. In: Perry O, Perry J, eds. Mathematics. London: Palgrave Macmillan, 1981:132-138.

6. Strasberg SM, Drebin JA, Mokadam NA, Green DW, Jones KL, Ehlers JP, et al. Prospective trial of a blood supply-based technique of pancreaticojejunostomy: effect on anastomotic failure in the Whipple procedure. J Am Coll Surg 2002;194:746-758; discussion 759-760. 